\title{
Metastases can occur in cirrhotic livers with patent portal veins
}

\author{
Zaid Mahdii ${ }^{1 \dagger}$, Mark G. Ettel ${ }^{2+}$, Raul S. Gonzalez ${ }^{3}$, John Hart ${ }^{4}$, Lindsay Alpert ${ }^{4}$, Jiayun Fang ${ }^{5}$, Natalia Liu ${ }^{5}$, \\ Suntrea T. Hammer ${ }^{6}$, Nicole Panarelli ${ }^{7}$, Jerome Cheng ${ }^{5}$, Joel K. Greenson ${ }^{5}$, Paul E. Swanson ${ }^{8}$ and Maria Westerhoff ${ }^{*}$
}

\begin{abstract}
Objectives: Metastases are common in non-cirrhotic livers but are considered unlikely in the setting of cirrhosis. However, the degree of fibrosis in cirrhosis may vary; thus metastases may still access the liver vasculature and present as a mass in cirrhotic livers. This possibility may affect pathologists' diagnostic algorithms when faced with a liver mass biopsy.
\end{abstract}

Methods: We hypothesized that metastases can occur in cirrhotic livers if fibrous remodeling is not severe or abnormal veno-arterial shunting exists to override an obstructed portal system. We searched departmental archives for cirrhotic livers with masses, categorizing fibrosis by Laennec staging: $4 \mathrm{~A}=$ mild cirrhosis, $4 \mathrm{~B}=$ moderate, $4 \mathrm{C}=$ severe.

Results: Of 1453 cirrhotic livers with masses, 1429 were primary tumors and 24 were metastases (1.7\%). Of livers with metastases, most had $4 \mathrm{~A}$ or $4 \mathrm{~B}$ cirrhosis by Laennec staging $(n=17 ; 71 \%)$. Eleven patients were evaluated by ultrasound Doppler; 2 of 5 with Laennec $4 \mathrm{C}$ had reversal of portal vein flow, but all 4A \& 4B patients had patent portal veins without reversed flow. Echocardiograms (13 patients) showed no ventricular or atrial septal defects or arteriovenous shunts.

Conclusions: Metastases are uncommon in cirrhotic livers, accounting for $1.7 \%$ of masses. Most involved livers had mild or moderate cirrhosis (Laennec 4A/4B) and patent portal veins; however, as some Laennec $4 \mathrm{C}$ cases also contained metastases, obstructed portal access may not be enough to deter metastatic access.

Keywords: Liver, Metastases, Liver mass, Laennec staging, Cirrhosis

\section{Introduction}

The liver is among the most common sites of metastatic disease. However, while metastases are common in noncirrhotic livers, multiple autopsy studies have confirmed that they are rare in the setting of cirrhosis.[1-3] As such, pathologists generally regard metastatic malignancy as an unlikely diagnosis when evaluating biopsies from mass lesions in cirrhotic livers.

\footnotetext{
* Correspondence: mwesterh@med.umich.edu Zaid Mahdi and Mark G. Ettel are co-first authors.

${ }^{5}$ Department of Pathology, University of Michigan, Faculty Suite Rm. 36-1221-65 2800 Plymouth Rd, Building 35, 48109 Ann Arbor, MI, USA Full list of author information is available at the end of the article
}

To explain the frequency of liver metastases in the general population and the contrasting rarity of metastases in the cirrhotic liver, a review of the main hypotheses for development of metastatic disease is appropriate. The "seed and soil" hypothesis, first offered by Stephen Paget in 1889, states that the "seed" of metastatic tumor cells may develop into a full-fledged metastasis only once it reaches the favorable "soil" of a hospitable environment such as the otherwise healthy liver.[4, 5] In contrast, the advanced fibrosis and microenvironment of the cirrhotic liver may be an "unfavorable soil" for metastatic carcinomas. Alternatively, James Ewing hypothesized that hemodynamic factors are responsible for the

(c) The Author(s). 2021 Open Access This article is licensed under a Creative Commons Attribution 4.0 International License, which permits use, sharing, adaptation, distribution and reproduction in any medium or format, as long as you give appropriate credit to the original author(s) and the source, provide a link to the Creative Commons licence, and indicate if changes were made. The images or other third party material in this article are included in the article's Creative Commons licence, unless indicated otherwise in a credit line to the material. If material is not included in the article's Creative Commons licence and your intended use is not permitted by statutory regulation or exceeds the permitted use, you will need to obtain permission directly from the copyright holder. To view a copy of this licence, visit http://creativecommons.org/licenses/by/4.0/ The Creative Commons Public Domain Dedication waiver (http://creativecommons.org/publicdomain/zero/1.0/) applies to the data made available in this article, unless otherwise stated in a credit line to the data. 
appearance and distribution of metastases.[4] In noncirrhotic livers, carcinomas may access the liver through the dominant portal venous blood supply, resulting in a high incidence of liver metastases from gastrointestinal primary malignancies. However, in cirrhosis, there is scarring and increased intrahepatic resistance to blood flow. It is plausible that this scarring may deter the entry of metastases via reversal of portal venous blood flow and development of porto-systemic shunts that bypass the liver.[6, 7] Previous studies show that the hepatic artery is another route for metastatic cells to access the liver. $[8,9]$.

Contrary to previous dogma, cirrhosis is not an "all or nothing," irreversible process. There can be a spectrum of fibrosis, and the extent of fibrosis in a given patient likely will change (towards either end of the spectrum) over time. The Laennec staging classification further categorizes cirrhosis into mild, moderate, and severe fibrosis and correlates with clinical outcomes, including decompensation and liver-related death.[10] Since the altered blood flow in cirrhosis is likely due (at least in part) to fibrosis, the degree of fibrosis in cirrhosis may affect the ability of in-transit metastases to access the liver vasculature.[7] Hence, although we do not use Laennec staging in daily diagnostic practice, we employed it for the purposes of this study, hypothesizing that metastases can occur in cirrhotic livers where the fibrous remodeling is not severe. As an extension of this argument, we also hypothesized that abnormal venoarterial shunts may effectively bypass more severe stages of fibrosis. While there are robust autopsy-based studies examining the prevalence of metastases in cirrhotic livers, studies of metastases in the cirrhotic liver of living patients are essentially limited to colorectal cancer.[6, 11-13] With that in mind, and given that liver masses are a common and critically important specimens in the practice of surgical pathologists, we aim in this study to provide a systematic evaluation of the likelihood that a biopsy or resections of a mass in cirrhotic liver will contain metastatic disease.

\section{Materials and methods}

\section{Cases and Data Collection}

We searched the electronic pathology records at 7 tertiary care institutions for biopsy, excision, or surgical resection specimens of cirrhotic livers with mass lesions. Among these specimens were well-defined examples of metastases to liver. As our control population, we selected cases from surgical pathology archives at these institutions of cirrhotic livers without mass lesions in patients who had histologic confirmation of distant metastases at other sites. Because few such control cases were identified in our surgical pathology archives, autopsy archives were also searched. We also developed separate control populations of patients with primary liver neoplasms and cirrhosis, and patients with masses in non-cirrhotic livers. Patient medical records of cirrhotic metastasis cases were reviewed for clinical history (including possible sequelae of cirrhosis), radiologic data (including echocardiogram and ultrasound Doppler results, which were reviewed for evidence of veno-arterial shunting) and laboratory values. This information was also reviewed in the controls with cirrhosis and metastatic disease that did not involve the liver, and in a subset consisting of $10 \%$ of the controls with primary liver tumors and cirrhosis. The study was approved by the Institutional Review Boards at each of the involved institutions.

\section{Special Stains and Immunohistochemistry}

Routine hematoxylin and eosin and trichrome stains for case and control specimens were reviewed, when available, in all study cases and controls. For those cases where a trichrome stain was not available, new Masson or Gomori trichrome stains were performed according to standard institutional protocols at each study site. Immunohistochemical stains for CD34 were performed on all specimens from cases of metastases in cirrhosis and in the subset of $10 \%$ of primary liver cancers chosen for further clinical review, again using routine protocols at each institution.

\section{Histologic Review}

Cirrhosis was categorized by the Laennec fibrosis staging as described previously: $4 \mathrm{~A}=$ mild cirrhosis (delicate fibrous septa seen on trichrome stain), $4 \mathrm{~B}=$ moderate (at least 2 broad septa), $4 \mathrm{C}$ = severe cirrhosis (at least 1 very broad septum or many minute nodules) (Fig. 1).[10] Immunohistochemical staining for aberrant CD34 in the sinusoids in the background cirrhotic liver was considered evidence of sinusoidal capillarization due to severe portal vein obstruction.

\section{Results}

Of the 1453 biopsies, excisions, or resections of masses in cirrhotic livers, $24(1.7 \%)$ were metastases (Table 1); representative examples of metastases are seen in Fig. 2. The clinicopathologic features of these metastases are summarized in Table 2. The average age of patients with metastases in cirrhotic was 64 years (range 47-78); most (15) patients were women. The most common primary tumor responsible for metastases in cirrhotic livers was colorectal carcinoma (8 cases; $33 \%$ ). Other primary tumors included neuroendocrine neoplasms from any site (5; $21 \%)$, as well as carcinomas of pancreas (4; 17\%), upper gastrointestinal tract $(2 ; 8 \%)$, breast $(2 ; 8 \%)$, fallopian tube $(1 ; 4 \%)$ and lung $(1 ; 4 \%)$. A single case $(4 \%)$ of carcinoma of unknown primary was included. 


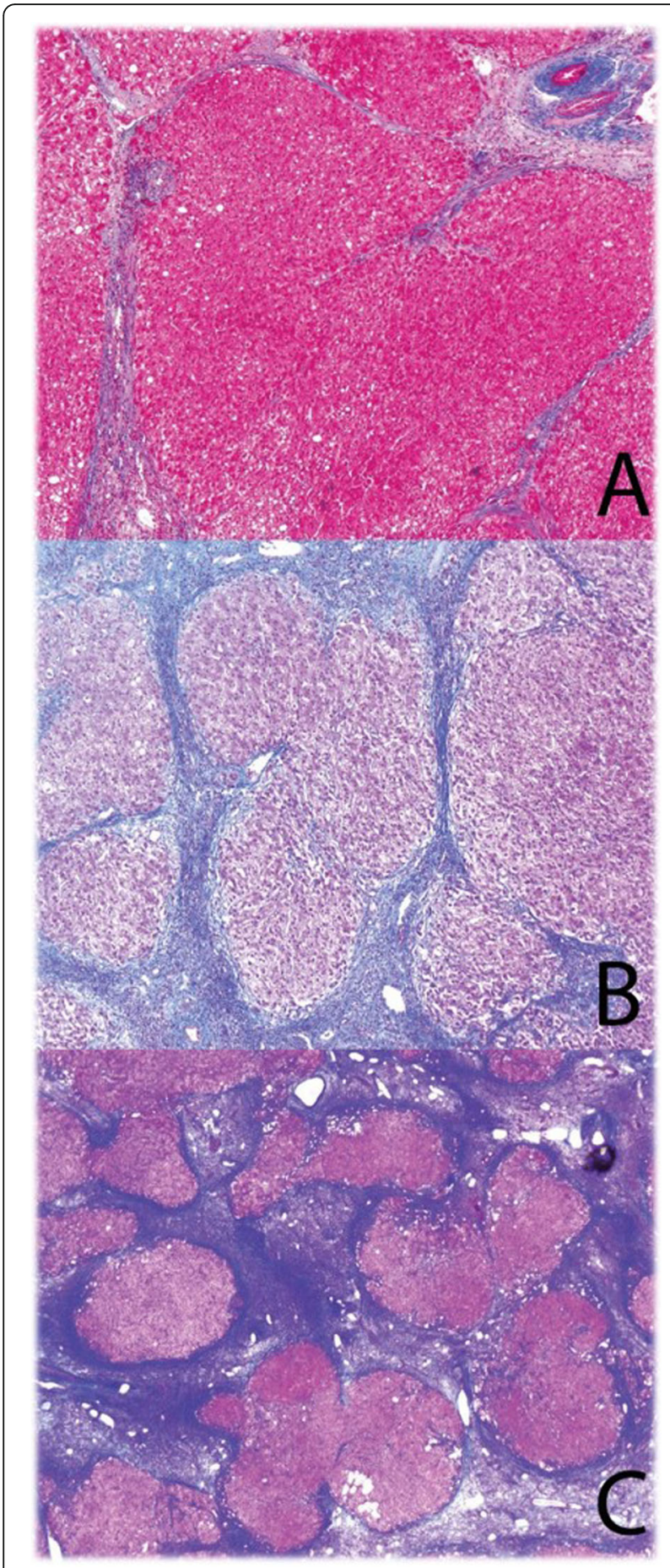

Fig. 1 Laennec staging in cirrhosis. a Laennec stage 4A is characterized by mild cirrhosis with delicate fibrous septae, trichrome stain. b Laennec stage 4B (moderate) cirrhosis with broad fibrous septae, trichrome stain. c Laennec 4 C (severe) cirrhosis with very broad septae, trichrome stain.
Neuroendocrine neoplasms were from pancreas (2), small bowel (1), lung (1) or from an unknown site (1).

For comparison, we identified 8 liver samples (including 5 autopsy specimens) in patients who had both cirrhotic livers and malignancies with distant metastases at other sites without involvement of the liver. The average age of patients in this control group was 63 years (range $52-78) ; 5$ of these patients were women. The primary sites in this control population included carcinomas from lung (1), colon (1), fallopian tube (1), breast (1), renal (1), pancreas (1), and thyroid (1), as well as 1 poorly differentiated neoplasm which was thought to be a malignant mesothelioma. We also examined 147 primary liver tumors from cirrhotic livers (138 hepatocellular carcinomas, 9 cholangiocarcinomas). Finally, we identified 29 metastases to non-cirrhotic livers (7 surgical excisions/resections and 22 biopsies). Of these, 5 (3 biopsies, 2 excisions/resections) had fibrosis patterns up to and including bridging fibrosis, insufficient for a diagnosis of cirrhosis. The primary sites of carcinomas in this control group included colon (11), esophagus (3), lung (3), pancreas (2), breast (2), prostate (1), ureter (1), bladder (1), kidney (1), and pituitary (1), Three melanomas were also included.

\section{Histologic evaluation of metastases in cirrhotic livers and controls}

By Laennec staging, most (17 of 24) cirrhotic livers with metastases were $4 \mathrm{~A}(10)$ or $4 \mathrm{~B}(7)$, while the remaining 7 livers were Laennec $4 \mathrm{C}$ (Fig. 3). The majority of samples in the control cirrhosis group from patients with malignancies that had metastasized to sites other than liver were also Laennec 4A (3) or 4B (3), while only 2 livers were Laennec $4 \mathrm{C}$. Of the cirrhotic livers with primary liver tumors, 64 were Laennec $4 \mathrm{~A}$ and 64 were $4 \mathrm{~B}$. The remaining 28 cases were $4 \mathrm{C}$.

CD34 immunohistochemical tests did not show evidence of generalized sinusoidal capillarization in any cases of metastases in cirrhotic livers. In comparison, 1 of 14 controls with primary liver cancer in cirrhosis had CD34 positivity in the background liver suggestive of generalized sinusoidal capillarization.

\section{Clinical, radiologic and laboratory data in cirrhotic livers and controls}

The causes of cirrhosis in patients with metastases were steatohepatitis (13 total cases of steatohepatitis; 5 of which were non-alcoholic, 4 of which were alcoholic, and 4 of which were due to steatohepatitis not otherwise specified), hepatitis $C$ virus (7 cases), and cryptogenic (4 cases). There was no discernible relation between etiology of cirrhosis and the primary tumor responsible for metastasis; each etiology was seen in association with multiple sites of origin for metastases. Mean albumin 


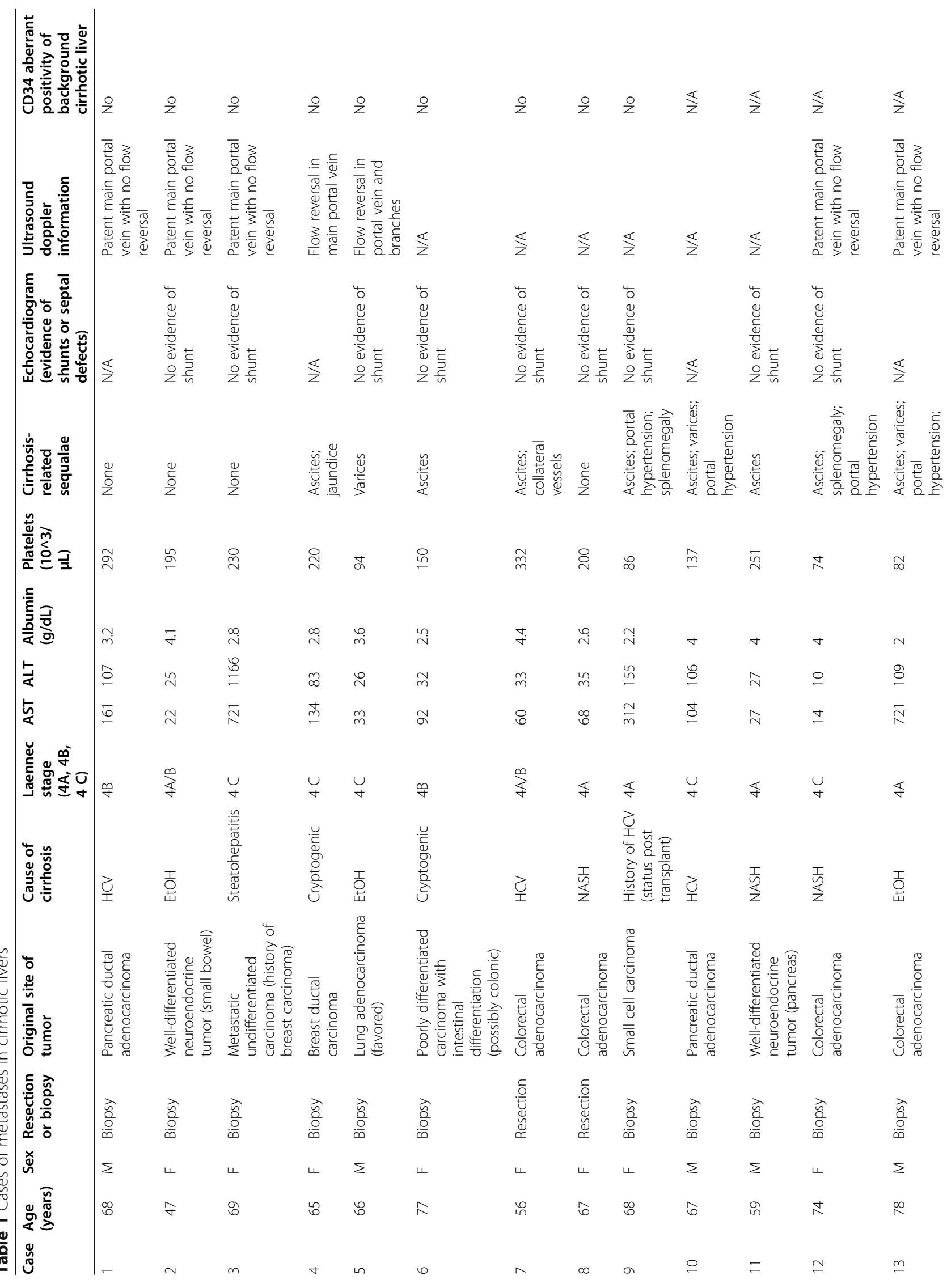




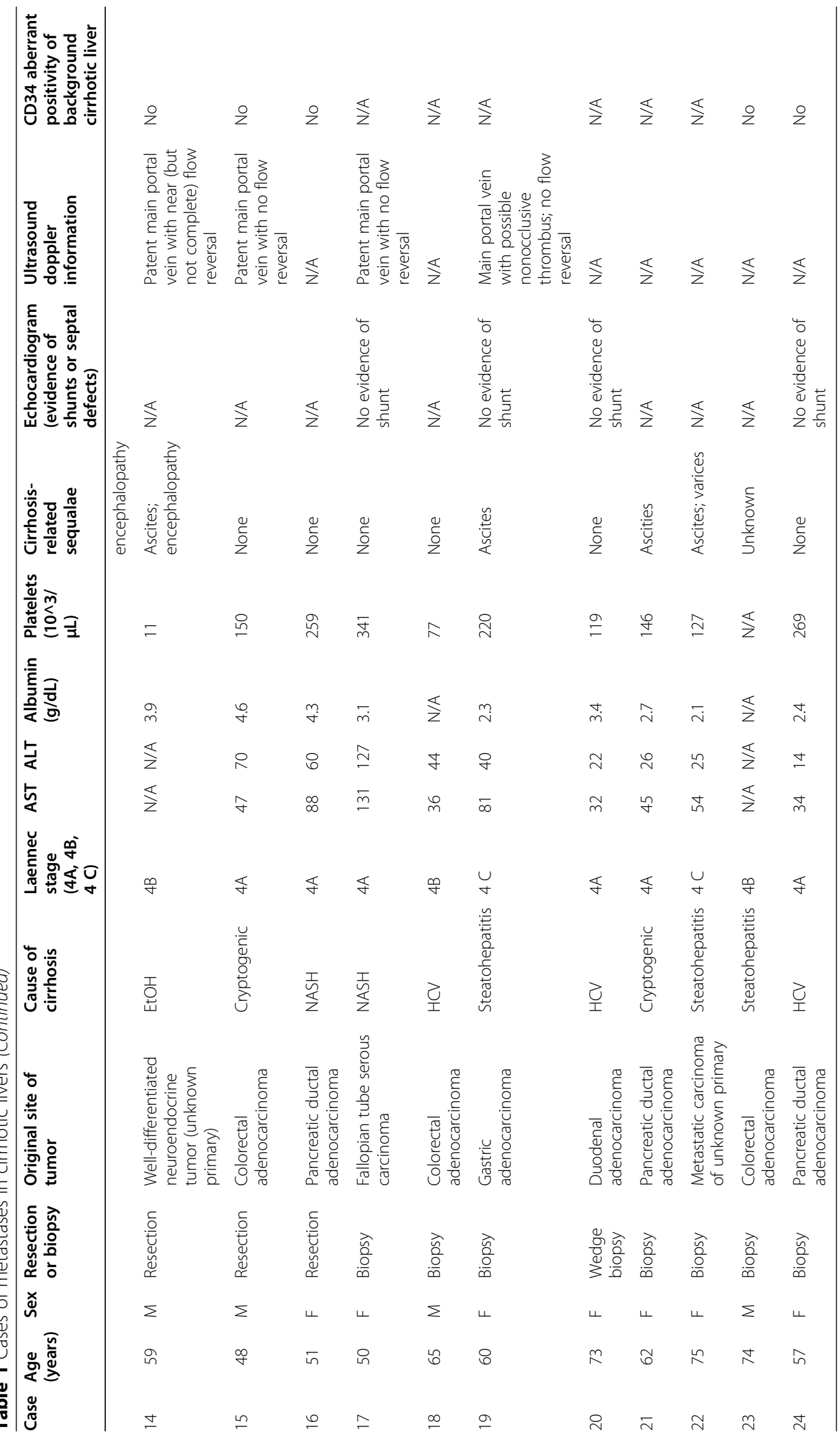




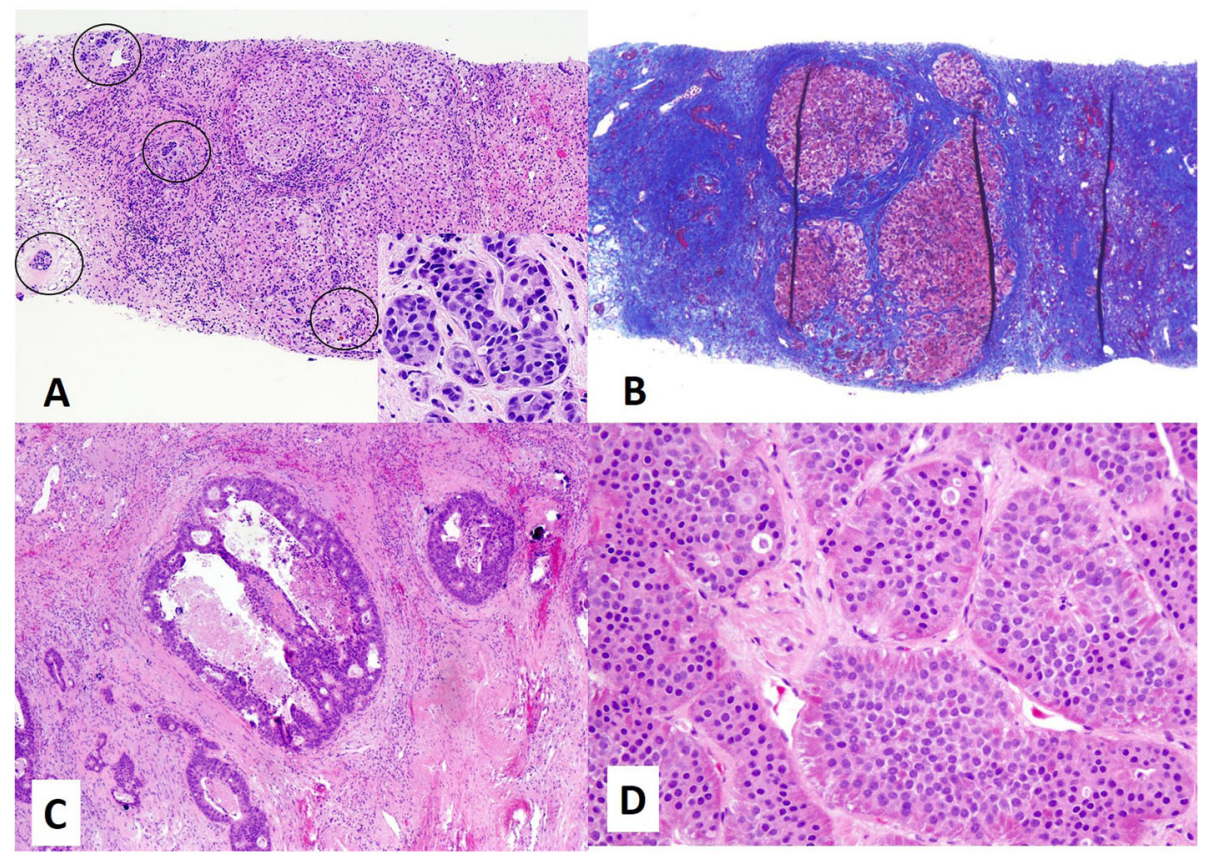

Fig. 2 Representative metastases in cirrhotic livers. a Metastatic invasive ductal carcinoma of breast, within cirrhotic parenchyma, H\&E. The circled areas are the metastatic tumor cells. Inset shows a high power magnification of the carcinoma. $\mathbf{b}$ Trichrome stain of the case of metastatic invasive ductal carcinoma, demonstrating cirrhosis. c Metastatic colorectal adenocarcinoma, H\&E. d Metastatic well-differentiated neuroendocrine tumor, H\&E.

values were $3.2 \mathrm{~g} / \mathrm{dL}$ in patients with metastases and cirrhotic livers, with mildly elevated transaminase levels (mean aspartate aminotransferase (AST) level $=137$; mean alanine aminotransferase level $($ ALT $)=106)$. Mean platelet count was $177 \times 10^{\wedge} 3 / \mathrm{uL}$ in these patients (Table 1). In the control population of cirrhotic patients with malignancies that metastasized to sites other than the liver, mean albumin values were $2.9 \mathrm{~g} / \mathrm{dL}$ with mean transaminase values $62 \mathrm{AST}$ and $30 \mathrm{ALT}$, while average platelet count was $161 \times 10^{\wedge} 3 / \mathrm{uL}$.

Clinically, 13 of 24 patients with metastases in cirrhotic livers had sequelae of decompensated cirrhosis, including ascites, varices or other evidence of portal hypertension, and jaundice; ascites was the most common sequela and was present in 12 patients. Ultrasound Doppler imaging was available for 11 patients and showed reversal of portal vein flow in 2 of 5 patients with Laennec $4 \mathrm{C}$ cirrhosis, but all $4 \mathrm{~A}$ and $4 \mathrm{~B}$ patients had patent portal veins without evidence of reversed flow. In addition, portal vein thrombi were suggested via Doppler or other imaging in 3 additional Laennec $4 \mathrm{C}$ patients; overall only 2 of 7 patients with Laennec $4 \mathrm{C}$ cirrhosis had patent portal veins and normal flow. Echocardiogram results were available for 13 patients, including 4 with Laennec $4 \mathrm{C}$ cirrhosis, and did not show ventricular or atrial septal defects or arteriovenous shunts (Table 1).
In the control population of patients with distant metastases at other sites without involvement of the liver, 5 of 8 patients had sequelae of cirrhosis, including ascites and portal hypertension. Two patients in this group were evaluated by ultrasound Doppler imaging; the portal vein was patent in both and no reverse flow was seen. Echocardiogram results were available for 7 patients, including one patient with Laennec $4 \mathrm{C}$ cirrhosis; one patient had a patent foramen ovale with a low-grade left-right shunt, while the remaining patients had neither ventricular or atrial septal defects nor shunting.

12 of 14 patients with primary liver cancer in cirrhosis had cirrhosis-related sequelae. Of the 10 cases of primary liver cancer in cirrhosis where Doppler data was reviewed, 1 patient had a right to left shunt while the remaining patients had no significant abnormality on imaging.

\section{Discussion}

Our study offers a multifaceted approach to better elucidate the phenomenon of metastasis to the cirrhotic liver. We combined histologic features with vascular dynamics in order to shed light on the physiology of metastasis to the cirrhotic liver and delinieate clinical scenarios in which this uncommon occurrence may be present. We found that even advanced fibrosis is not an absolute barrier to metastatic disease, particularly if portal 
Table 2 Summary of clinicopathologic features of metastases in cirrhosis

\begin{tabular}{|c|c|}
\hline & $\begin{array}{l}n(\%) \text { unless stated } \\
\text { otherwise }\end{array}$ \\
\hline \multicolumn{2}{|l|}{ Sex } \\
\hline Female & $15(62.5)$ \\
\hline Male & $9(37.5)$ \\
\hline Age, mean (range) & $64(47,78)$ \\
\hline \multicolumn{2}{|l|}{ Laennec Stage } \\
\hline $4 \mathrm{~A}$ & $10(41.7)$ \\
\hline $4 \mathrm{~B}$ & $7(29.2)$ \\
\hline $4 C$ & $7(29.2)$ \\
\hline \multicolumn{2}{|l|}{ Primary site } \\
\hline Colorectal & $8(33)$ \\
\hline Neuroendocrine & $5(21)$ \\
\hline Pancreas & $4(17)$ \\
\hline Upper Gastrointestinal & $2(8)$ \\
\hline Breast & $2(8)$ \\
\hline Mullerian & $1(4)$ \\
\hline Lung & $1(4)$ \\
\hline Unknown & $1(4)$ \\
\hline$A S T$, mean $\pm S D$ & $137 \pm 200$ \\
\hline$A L T$, mean $\pm S D$ & $106 \pm 240$ \\
\hline Albumin, mean $\pm S D$ & $3.2 \pm 0.8$ \\
\hline Platelets, mean $\pm S D$ & $177 \pm 89$ \\
\hline Cirrhosis-related sequelae & $13(54.2)$ \\
\hline $\begin{array}{l}\text { Echocardiogram with evidence of } \\
\text { shunt }\end{array}$ & $0(0)$ \\
\hline Reversal of portal vein flow & $2(18.2)$ \\
\hline
\end{tabular}

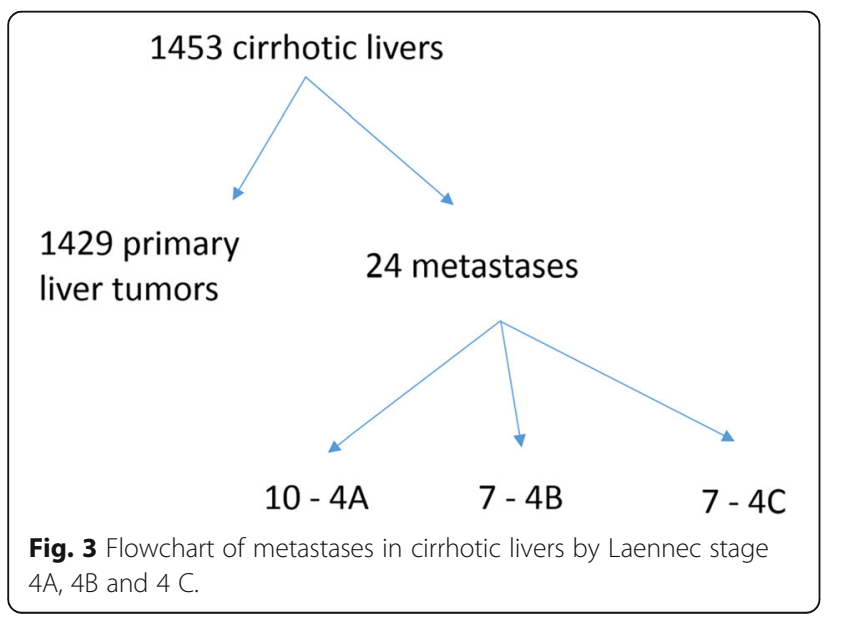

circulation is intact and if sinusoidal capillarization, as demonstrated by negative CD34 imunostains, is absent. Although normally, pathologists and clinicians may not expect metastases in a cirrhotic liver, we conclude that involvement from extrahepatic primary tumors is still a possibility, particularly in these settings.

Numerous autopsy studies have demonstrated the rarity of metastases in cirrhotic livers, with prevalence ranging $0-2.9 \%$ in these studies, although systematic and complete clinical cohort studies in living patients are largely lacking (Table 3).[1-3, 14-20] Our results confirm that metastases are rare in cirrhotic livers and provide an estimate of the prevalence of this phenomenon from the perspective of the practicing surgical pathologist. Of 1453 biopsies, excisions or resections of cirrhotic livers with mass lesions, only $1.7 \%$ contained metastases from extrahepatic malignancies.

Interestingly, the distribution of metastases in cirrhosis is similar to that seen in liver metastases as a whole; a nationwide study of all histologically confirmed liver metastases in the Netherlands showed that colorectal carcinoma made up $35 \%$ of overall metastases compared to $33 \%$ in our study.[21] Our study of cirrhotic livers had proportionately more pancreas and upper gastrointestinal primary tumors $(38 \%)$ compared to the $18 \%$ of overall metastases in the Netherlands study, although given the relatively small number of metastases in our study it is difficult to say that these results represent a true difference. With our finding that 16 of $24(67 \%)$ metastases are from primary malignancies other than colorectal carcinoma, we establish that while the colorectum may be the most common single site responsible for metastasis in this setting, most metastases are from primaries outside the colorectum. Thus a wide differential diagnosis is essential in those rare cases where a metastasis is suspected in a cirrhotic liver.

Several rationales have been offered for the rarity of metastases in cirrhotic livers. Since Paget originally offered his seed-and-soil hypothesis, evidence has emerged supporting the metastasis-suppressing role of various factors in the cirrhotic microenvironment. One proposed explanation for the rarity of metastases in cirrhotic livers is that chronic liver disease reduces the function of hepatocyte membrane lectins, which otherwise may promote metastases by recognizing tumor cell surface carbohydrates and adhering to tumor cells.[22-24] Supporting this hypothesis, injection of hepatocyte lectin blocking agents in a mouse model inhibited liver metastases without any effect on number or size of lung metastases.[22] However, blockades of hepatocyte lectins are likely nonspecific unless glycosylation patterns of these lectins are unique, and liver sinusoidal endothelial cell lectins have also been show to play a role in tumor cell adherence.[25] Other possible factors in the low prevalence of 
Table 3 Previous studies of metastases in patients with cirrhotic livers

\begin{tabular}{|c|c|c|c|c|c|}
\hline Reference & $\begin{array}{l}\text { Publication } \\
\text { Year }\end{array}$ & $\begin{array}{l}\text { Overall } \\
\text { autopsies }\end{array}$ & $\begin{array}{l}\text { Autopsies with } \\
\text { cirrhosis }\end{array}$ & $\begin{array}{l}\text { Autopsies with cirrhosis and } \\
\text { extrahepatic cancer }\end{array}$ & $\begin{array}{l}\text { Autopsies with metastases in } \\
\text { cirrhotic livers }\end{array}$ \\
\hline Lisa et al[16] & 1942 & 6,036 & & 22 & 6 \\
\hline Wallach et al[3] & 1953 & 10,156 & 441 & 67 & 3 \\
\hline Lieber[1] & 1957 & 29,779 & 1,073 & 133 & 6 \\
\hline Gall[14] & 1960 & 1,001 & 682 & 108 & 29 \\
\hline $\begin{array}{l}\text { Ruebner } \\
\text { et al[19] }\end{array}$ & 1961 & 23,000 & 399 & 54 & 11 \\
\hline Norkin et al[18] & 1962 & 15,713 & 1,268 & 121 & 37 \\
\hline Goldstein[15] & 1969 & 4,166 & & 31 & 12 \\
\hline Melato et al[17] & 1989 & 11,752 & 1,029 & 160 & 29 \\
\hline $\begin{array}{l}\text { Vanbockrijck } \\
\text { et al[20] }\end{array}$ & 1992 & 2,162 & 153 & 30 & 10 \\
\hline $\begin{array}{l}\text { Pereira-Lima } \\
\text { et al[2] }\end{array}$ & 2003 & 7,092 & 111 & 6 & 0 \\
\hline Summary & & 110,857 & 5,156 & 732 & 143 \\
\hline
\end{tabular}

metastases in patients with cirrhosis include microRNA let-7a cluster involved in regulating the epithelialmesenchymal transition of colorectal carcinoma, regulation of FasR expression, and matrix metalloproteinase activity.[26] Thus, hepatocytes, sinusoidal cells, and Kupffer cells all may have a role in the "soil" of the cirrhotic microenvironment. As our study shows that metastases still occur in cirrhosis especially in those patients with less severe fibrosis, further research into the effects of cirrhosis on these microenvironmental factors may be worth pursuing.

With respect to Ewing's hemodynamic hypothesis, the portal venous system is presumed to be the usual route of entry for metastases into the liver. In cirrhosis, architectural distortion and increased vascular resistance in the portal system contribute to portal hypertension. Porto-systemic shunts develop in response to this increase in portal pressure.[7] This set of vascular phenomena is thought to act against a portal route for metastasis of colorectal and upper gastrointestinal carcinomas. Interestingly, most patients in our cohort, including all with Laennec stage $4 \mathrm{~A}$ and $4 \mathrm{~B}$ cirrhosis, had patent portal veins, although most patients with Laennec stage $4 \mathrm{C}$ cirrhosis showed portal venous thrombi or reversal of portal flow. Thus, while the severe hemodynamic changes of Laennec $4 \mathrm{C}$ may make metastasis less likely, it is still possible to develop metastasis in the setting of reversed portal blood flow. Given that hepatic artery resistance is decreased and hepatic artery velocity is increased in cirrhosis as a buffer response to increased portal resistance, we suspect that arterial entry may play a role in these cases.[7].

Finally, in addition to hypotheses related to the physiology and microenvironment of the cirrhotic liver, it has been suggested that the rarity of metastases in cirrhosis may ultimately be a consequence of significant comorbidity: patients with both cirrhosis and extrahepatic cancer may simply not live long enough to develop metastases. Co-occurrence of cirrhosis and extrahepatic cancer is rare even without liver metastasis. $[1,3,16]$ Furthermore, in one comparison of colorectal carcinoma patients with cirrhosis compared to similar patients without cirrhosis, overall survival was lower in cirrhotic patients despite similar progression-free survival.[27] In our study, both patients with metastases in cirrhosis and the control group of patients with cirrhosis and nonliver metastasis are composed predominantly of patients with Laennec $4 \mathrm{~A}$ or $4 \mathrm{~B}$ cirrhosis. While other factors likely contribute to the rarity of metastases in patients with Laennec 4 C cirrhosis, the overall metabolic burden of both severe cirrhosis and metastatic malignancy may also play a role.

One additional possibilility worth considering is the timeline of progression to cirrhosis compared with the rate of progression of metastatic disease. Our data do not indicate any obvious relation between etiology of cirrhosis and the primary site responsible for metastatic disease. However, it is known that some diseases progress to cirrhosis faster than others, while some cancers grow faster or slower than others. Thus, it is possible that some cases of apparent metastases in cirrhotic livers arise when indolent, slow-growing neoplasms metastasize to livers that are non-cirrhotic at the time of metastasis, after which fibrosis progresses to cirrhosis and metastasis is discovered at a later date.

A strength of our study is a new approach to the topic of metastases in cirrhotic livers. It draws on the prevalence and pathologic characteristics of these cases from the perspective of surgical pathologic evaluation rather than autopsies. In addition, because our study design 
allowed us to both review imaging data and evaluate the CD34 expression patterns in the sinusoidal endothelium of cirrhotic tissue, we were able to use a comprehensive approach to evaluate vascular status of cirrhotic patients with metastases.

Our study does have important limitations. While our analysis provides an overview of cirrhotic metastases in terms of lesions which may be biopsied or resected for pathologic evaluation, our ability to determine overall prevalence and epidemiology is limited by the clinical situations in which samples might be procured. In patients with cirrhosis and multiple metastases in both the liver and other organs, clinicians may opt for a less technically challenging biopsy in another organ, falsely reducing the prevalence of metastases in our study. In addition, the retrospective nature of our analysis and the chart review design limit the availability of certain clinical data, such as imaging, which were not available on all patients. Finally, the evaluation of Laennec stage in some of the core biopsy specimens is limited by the possible confounding effect of the target mass on adjacent liver parenchyma. To help mitigate this influence, cases where mass effect could not be obviously differentiated from cirrhosis were excluded from our study. Notably, in our control group of noncirrhotic patients with metastases, we did not identify any biopsies with sufficient fibrosis to otherwise meet the criteria for cirrhosis, suggesting that 'mass effect' likely does not mimic advanced stage liver disease in most cases. We, nonetheless, demonstrate that metastases do occur in the cirrhotic liver, albeit rarely, and display a predilection for the less severe end of the cirrhotic spectrum. Thus, secondary liver involvement by extrahepatic malignancy remains in the differential diagnosis of liver masses from patients with cirrhosis.

\section{Abbreviations}

AST: Aspartate aminotransferase; ALT: Alanine aminotransferase

\section{Acknowledgements}

Not applicable.

\section{Authors' contributions}

All authors contributed to the study conception and design. Material preparation, data collection and analysis were performed by Zaid Mahdi, Mark G. Ettel and Maria Westerhoff. The first draft of the manuscript was written by Zaid Mahdi and Mark Ettel (co-first authors) and all authors commented on previous versions of the manuscript. All authors read and approved the final manuscript.

\section{Funding}

No outside funding received.

\section{Availability of data and materials}

The datasets generated during and/or analysed during the current study are available from the corresponding author on reasonable request.

\section{Ethics approval}

Approved by IRB at each participating institution.
Consent for publication

Not applicable

\section{Competing interests}

The authors declare that they have no conflict of interest.

\section{Consent to participate}

Not applicable.

\section{Code availability}

The code used during the current study are available from the corresponding author on reasonable request.

\section{Author details}

'Department of Pathology, Emory University School of Medicine, Atlanta, GA, USA. ${ }^{2}$ Department of Pathology, University of Rochester Medical Center, Rochester, NY, USA. ${ }^{3}$ Department of Pathology, Beth Israel Deaconness Medical Center, Boston, MA, USA. ${ }^{4}$ Department of Pathology, University of Chicago, Chicago, IL, USA. ${ }^{5}$ Department of Pathology, University of Michigan, Faculty Suite Rm. 36-1221-65 2800 Plymouth Rd, Building 35, 48109 Ann Arbor, MI, USA. ${ }^{6}$ Department of Pathology, University of Texas Southwestern Medical Center, Dallas, TX, USA. ${ }^{7}$ Department of Pathology, Montefiore Medical Center, Bronx, NY, USA. ${ }^{8}$ Department of Pathology, University of Washington, Seattle, WA, USA.

Received: 9 December 2020 Accepted: 10 February 2021

Published online: 27 February 2021

\section{References}

1. Lieber MM. The rare occurrence of metastatic carcinoma in the cirrhotic liver Am. J Med Sci. 1957;233:145-52.

2. Pereira-Lima JE, Lichtenfels E, Barbosa FS, Zettler CG, Kulczynski JM. Prevalence study of metastases in cirrhotic. livers Hepatogastroenterology. 2003;50:1490-5.

3. Wallach JB, Hyman W, Angrist AA. Metastasis to liver portal cirrhosis Am. J Clin Pathol. 1953;23:989-93.

4. Liu Q, Zhang H, Jiang X, Qian C, Liu Z, Luo D. Factors involved in cancer metastasis: a better understanding to "seed and soil" hypothesis Mol. Cancer. 2017;16:176. doi:https://doi.org/10.1186/s12943-017-0742-4.

5. Paget $\mathrm{S}$. The distribution of secondary growths in cancer of the breast. Cancer Metastasis Rev. 1889:8:98-101.

6. Cai B, Liao K, Song XQ, Wei WY, Zhuang Y, Zhang S. Patients with chronically diseased livers have lower incidence of colorectal liver metastases: a meta-analysis. PLoS One. 2014;9:e108618. doi:https://doi.org/1 0.1371/journal.pone.0108618.

7. Iwakiri Y, Shah V, Rockey DC. Vascular pathobiology in chronic liver disease and cirrhosis - current status and future directions. J Hepatol. 2014;61:91224. doi:https://doi.org/10.1016/j.jhep.2014.05.047.

8. Ackerman NB (1972) Experimental studies on the circulation dynamics of intrahepatic tumor blood supply Cancer 29:435-439. doi: 10.1002/10970142(197202)29:2<435::aid-cncr2820290227>3.0.co;2-k

9. Ridge JA, Bading JR, Gelbard AS, Benua RS, Daly JM (1987) Perfusion of colorectal hepatic metastases. Relative distribution of flow from the hepatic artery and portal vein Cancer 59:1547-1553. doi: https://doi.org/10.1002/ 1097-0142(19870501)59:9<1547::aid-cncr2820590903>3.0.co;2-6

10. Kim SU, Oh HJ, Wanless IR, Lee S, Han KH, Park YN. The Laennec staging system for histological sub-classification of cirrhosis is useful for stratification of prognosis in patients with liver cirrhosis. J Hepatol. 2012;57:556-63. doi: https://doi.org/10.1016/j.jhep.2012.04.029.

11. Augustin G, Bruketa T, Korolija D, Milosevic M. Lower incidence of hepatic metastases of colorectal cancer in patients with chronic liver diseases:. meta-analysis Hepatogastroenterology. 2013;60:1164-8. doi:https://doi.org/1 0.5754/hge11561.

12. Chiou WY, Chang CM, Tseng KC, Hung SK, Lin HY, Chen YC, Su YC, Tseng CW, Tsai SJ, Lee MS, Li CY. Effect of liver cirrhosis on metastasis in colorectal cancer patients: a nationwide population-based cohort study Jpn. J Clin Oncol. 2015;45:160-8. doi:https://doi.org/10.1093/jjco/hyu178.

13. Kondo T, Okabayashi K, Hasegawa H, Tsuruta M, Shigeta K, Kitagawa Y. The impact of hepatic fibrosis on the incidence of liver metastasis from colorectal cancer Br. J Cancer. 2016;115:34-9. doi:https://doi.org/10.1038/ bjc.2016.155. 
14. Gall EA. Primary and metastatic carcinoma of the liver. Relationship to hepatic cirrhosis Arch. Pathol. 1960;70:226-32.

15. Goldstein MJ. Metastases in the cirrhotic liver N. Engl J Med. 1969;281:221. doi:https://doi.org/10.1056/nejm196907242810421.

16. Lisa JR, Solomon C, Gordon EJ. Secondary Carcinoma in Cirrhosis of the Liver Am. J Pathol. 1942;18:137-40.

17. Melato M, Laurino L, Mucli E, Valente M, Okuda K (1989) Relationship between cirrhosis, liver cancer, and hepatic metastases. An autopsy study Cancer 64:455-459. doi: https://doi.org/10.1002/1097-0142(19890715)64:2< 455::aid-cncr2820640219>3.0.co;2-c

18. Norkin SA, Heimann R, Fahimi HD (1962) Neoplasms, cirrhosis, and hepatic metastases Cancer 15:1004-1008. doi: https://doi.org/10.1002/10970142(196209/10)15:5<1004:aid-cncr2820150517>3.0.co;2-r

19. Ruebner BH, Green R, Miyai K, Caranasos G, Abbey H. The rarity of intrahepatic metastasis in cirrhosis of the liver. A statistical explanation with some comments on the interpretation of necropsy data Am. J Pathol. 1961; 39:739-46.

20. Vanbockrijck M, Kloppel G. Incidence and morphology of liver metastasis from extrahepatic malignancies to cirrhotic livers Zentralbl. Pathol. 1992;138: 91-6.

21. de Ridder J, de Wilt JH, Simmer F, Overbeek L, Lemmens V, Nagtegaal I. (2016) Incidence and origin of histologically confirmed liver metastases: an explorative case-study of 23,154 patients Oncotarget 7:55368-55376. doi: https://doi.org/10.18632/oncotarget.10552.

22. Beuth J, Ko HL, Oette K, Pulverer G, Roszkowski K, Uhlenbruck G. Inhibition of liver metastasis in mice by blocking hepatocyte lectins with arabinogalactan infusions and D-galactose J. Cancer Res Clin Oncol. 1987; 113:51-5.

23. Beuth J, Ko HL, Pulverer G, Uhlenbruck G, Pichlmaier H. Importance of lectins for the prevention of bacterial infections and cancer metastases Glycoconj. J. 1995;12:1-6. doi:https://doi.org/10.1007/bf00731862.

24. Uhlenbruck $\mathrm{G}$, Beuth J, Weidtman V. Liver lectins: mediators for metastases? Experientia. 1983;39:1314-5.

25. Zuo Y, Ren S, Wang M, Liu B, Yang J, Kuai X, Lin C, Zhao D, Tang L, He F. Novel roles of liver sinusoidal endothelial cell lectin in colon carcinoma cell adhesion, migration and in-vivo metastasis to. the liver Gut. 2013;62:116978. doi:https://doi.org/10.1136/gutinl-2011-300593.

26. Cheng L, Geng L, Dai B, Zheng T, Fu J, Qiao L, Cai W, Wang Y, Yang J. Repression of let-7a cluster prevents adhesion of colorectal cancer cells by enforcing a mesenchymal phenotype in presence of liver inflammation Cell. Death Dis. 2018;9:489. doi:https://doi.org/10.1038/s41419-018-0477-1.

27. Han EC, Ryoo SB, Park JW, Yi JW, Oh HK, Choe EK, Ha HK, Park BK, Moon SH, Jeong SY, Park KJ. Oncologic and surgical outcomes in colorectal cancer patients with liver cirrhosis: A propensity-matched study. PLoS One. 2017;12: e0178920. doi:https://doi.org/10.1371/journal.pone.0178920.

\section{Publisher's Note}

Springer Nature remains neutral with regard to jurisdictional claims in published maps and institutional affiliations.

Ready to submit your research? Choose BMC and benefit from:

- fast, convenient online submission

- thorough peer review by experienced researchers in your field

- rapid publication on acceptance

- support for research data, including large and complex data types

- gold Open Access which fosters wider collaboration and increased citations

- maximum visibility for your research: over $100 \mathrm{M}$ website views per year

At BMC, research is always in progress.

Learn more biomedcentral.com/submissions 